\title{
In the future all accredited radiotherapy physicists should have a PhD
}

\author{
Jeremy Booth ${ }^{1} \cdot$ May Whitaker $^{2} \cdot$ Clive Baldock $^{3}$
}

Published online: 21 September 2020

(c) Australasian College of Physical Scientists and Engineers in Medicine 2020

\section{Introduction and overview: Clive Baldock, moderator}

An important component of a physicist's education and training to become a qualified medical physics specialist accredited in a particular specialty area is the acquisition of an appropriate postgraduate degree. A further important and compulsory component is the experience gained whilst working in an appropriate clinical environment. Professional bodies in the physical sciences and engineering in medicine, such as the Australasian College of Physical Scientists and Engineers in Medicine (ACPSEM), have over many years been responsible for the setting and maintaining of professional standards to ensure the safe and clinicafflly competent practice of medical physicists. This has evolved over the years to comply with legislative and regulatory requirements, international evidence and standards, and the care and safety needs of all patients and staff.

With many medical physics specialists entering their chosen profession with a doctoral qualification, or having gained one whilst working in a medical physics environment, it is timely for this Topical Debate to explore the academic level of attainment required to undertake the role of medical physics specialist in the accredited radiotherapy specialisation. ${ }^{1}$

Arguing for the Proposition is Jeremy Booth, PhD. A/Prof Booth is the Head of Medical Physics at Northern Sydney Cancer Centre and an Adjunct Associate Professor at the University of Sydney. A/Prof Booth studied his PhD at the University of Adelaide investigating the dosimetric effect of treatment uncertainties. He trained as a clinical Medical

Clive Baldock

cbaldock@uow.edu.au

1 Northern Sydney Cancer Centre, Royal North Shore Hospital, St Leonards, NSW 2065, Australia

2 Department of Radiation Oncology, Chris O’Brien Lifehouse, 119-143 Missenden Road, Camperdown, NSW 2050, Australia

3 Research and Innovation Division, University of Wollongong, Wollongong, NSW 2522, Australia
Physicist from 2001 at Royal Prince Alfred Hospital, before relocating to St Leonards in 2007 as the Deputy Head of Medical Physics at Royal North Shore Hospital and Head of Medical Physics from 2011. A/Prof Booth has maintained a strong interest in image-guided radiotherapy, and more recently adaptive radiotherapy and the application of artificial intelligence (AI) in health. He was the ACPSEM Boyce Worthley Award winner in 2015, won the ASTRO Basic Sciences Award in 2014, has more than 20 invited presentations and over 100 publications in refereed journals. A/ Prof Booth has led several world first clinical trials including MLC tracking for prostate and lung, and now leads an industry partnership for adaptive radiotherapy with Varian Medical Systems.

Arguing against the proposition is May Whitaker, determinedly and unashamedly PhD-less. Ms Whitaker became the Head of Physics and Engineering at Chris O'Brien Lifehouse in Sydney early in 2020. As the new HoPE, she aims to break the mould of the traditional technical expert as chief, and usher in a new era of the physicist as the technical leader with influence and presence throughout the greater hospital and healthcare setting. Using her background in finance, sales, marketing, communication and client presentation as a springboard for this vision, Ms Whitaker is slowly but surely increasing the visibility of the Physics and Engineering group through a careful public relations campaign, underpinned by over 20 years of Radiation Oncology Medical Physics experience in NSW, Victoria and Queensland. Ms Whitaker has served on the ACPSEM Board of Directors for three separate terms and firmly believes in giving back to the professional community. She has, and continues to, act as mentor, advisor, supervisor, educator and shoulder to cry on. She has published some papers, given some lectures, presented at various conferences and led or assisted with several clinical projects. Ms Whitaker firmly believes the role of the ROMP is changing, as the nature of radiation oncology and cancer treatment changes. Hence the

\footnotetext{
${ }^{1}$ Contributors to Topical Debates are selected for their knowledge and expertise. Their position for or against a proposition may or may not reflect their personal opinions.
} 
Jeremy Booth

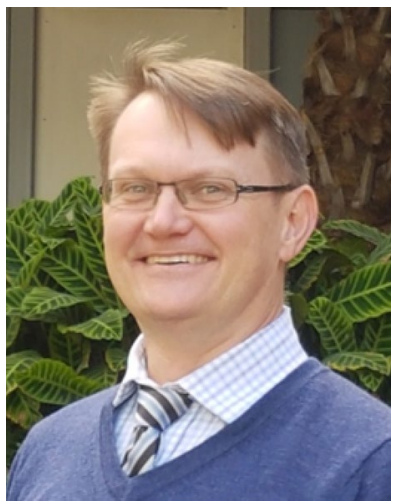

May Whitaker

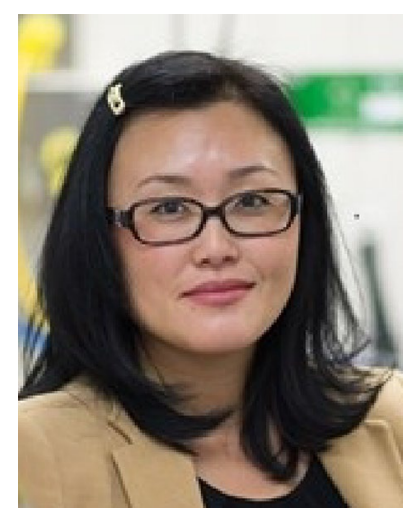

future-oriented ROMP must remain innovative and agile in order to stay relevant and valued.

\section{Opening statement-Jeremy Booth}

Radiotherapy is evolving as the world embraces automation in the everyday. Model-based treatment planning, artificial intelligence contouring, software automated patient quality assurance (QA) and artificial intelligence (AI) guided deformable image registration are all here and infiltrating [1]. The rapid uptake of these technologies, combined with the growth of privately owned and networked centres, is driving a "qualification creep" for radiotherapy physicists, which has been reported elsewhere in healthcare. I put to you that those with $\mathrm{PhDs}$ are very well prepared.

Let's first look at supply and demand. The introduction of the ACPSEM Training Education and Assessment Program (TEAP) in 2005, based on an extrapolated shortfall of linear accelerators (and physicists), has led to the funding of hundreds of physics registrars in 3-5 year contracts concluding at certification [2-6]. However, technology and business moves faster than government predictions. The green field cancer centre, often rural but also metropolitan, are overwhelmingly privately owned. These corporations successfully leverage networked expertise to operate with lower staff numbers offering the highest quality care. Government run cancer centres, with few exceptions, are slower to introduce labour reducing technology, but it will come. We need fewer radiotherapy physicists, yet have more. This will filter the best candidates and send others back to post-graduate education or other careers.

Let's also consider the phenomenon of "qualification creep", or "credentialism", where the entrance qualification for a vocation increases with time [7]. It has been well reported across healthcare, the key example from allied health being clinical psychology requiring a clinical doctorate. In radiotherapy physics, TEAP lifted the minimum up from a BSc, the qualification of a scientist, to an MSc [6]. In 2020, radiotherapy physics is evolving beyond technical support; in part because linear accelerators have evolved. Products like Varian Halcyon hint at a "black box" future where major medical devices will require only an annual audit certificate and nothing more. Meanwhile, physicists find themselves collaborating closer and more directly than ever with our (sub-specialised) radiation oncologist colleagues; adopting the language and skills of data scientists, programmers, inventors, grant-writers, and science communicators to drive innovation in clinical care.

It is the $\mathrm{PhD}$ that best teaches these skills. The $\mathrm{PhD}$ is a 3-4 year full time basic research degree designed specifically to teach the student how to undertake research independently. Fundamentally it requires problem solving, analysis, discussion and presentation. A PhD also teaches flexibility, adaptation to change and resilience. Core to discovering new ideas are the soft-skills of communicating them, garnering support, influencing and leading. A successful PhD is a master of the journal manuscript and the Powerpoint presentation, of international collaborations and the hallway conversation. They must present their ideas often, to experts and new audiences alike, taking new knowledge and moving fearlessly forward. This high level exchange prepares the $\mathrm{PhD}$ graduand for the workplace where the physicist will provide data to support-and debate-ideas with oncologists, therapists and administrators.

Radiotherapy physicists in the present will benefit from a $\mathrm{PhD}$, and those in the future will have one. It will be forced by supply and demand, and required by the evolving demands of our profession.

\section{Opening statement-May Whitaker}

Whilst a PhD is a worthy achievement it is not essential for accreditation and clinical work, hence it cannot be said that all future accredited physicists should have one. The PhD is inherently designed to facilitate a career in research or academia, and many find it hard to transfer those skills into a work environment [8]. Anecdotally, the disposition of $\mathrm{PhD}$ candidates may not be suited to routine clinical work [9]. 
The University of Sydney states that a $\mathrm{PhD}$ "will allow you to pursue research from one of a number of fields" [10]. In contrast, accreditation will allow you to pursue a broad, clinical, practical occupation.

In Australia and New Zealand, Radiation Oncology Medical Physicists (ROMPs) are responsible for the "safe operation and quality of systems in established medical radiation treatment machines ... and for the implementation and safety assessment of new treatment technologies" [11]. To become an accredited ROMP a physics graduate must undertake the TEAP as a medical physics registrar, a 3 to 5 year endeavour covering a range of topics from linear accelerator operation and quality assurance through to radiobiology and professional awareness.

At the early careers session of the EPSM national conference in 2016 keynote speaker, Dr Robert Jeraj, advised that if ground-breaking research and attainment of a $\mathrm{PhD}$ was the primary focus of one's career, a more fulfilling pathway would be to work for a dedicated research facility, rather than committing several years in TEAP to become an accredited, clinical ROMP. In a clinical department, spending a few inconsistent hours in between routine work will not result in a significant research output [12].

Instead, the accredited ROMP should focus on excelling in the clinical environment, understanding the theory and the rapidly changing tools, equipment, workflows and protocols that form the basis of radiotherapy, as well as remaining current with literature in all relevant areas. A flexible mindset to synthesise the sum of this knowledge and experience under high pressure, and communicate it effectively to the multidisciplinary team, is what makes an accredited ROMP the ideal clinical physicist.

Unfortunately, the scientific paradigm does not recognise clinical excellence. Instead, research output in terms of published papers and conference presentations are the yardsticks historically used to measure the success of a physicist. An accredited ROMP receives no accolades for performing essential quality assurance checks, recommending a treatment pathway for a complicated tumour or working long hours to ensure a patient's treatment plan can be safely and accurately delivered. Hence a $\mathrm{PhD}$ is often desired for the instant global recognition those three letters confer.

Research is an essential part of the TEAP program and accreditation status is contingent upon it. Indeed, without research the technologies and techniques used to treat cancer patients would never progress. However, for an accredited ROMP in a clinical department, the research skills developed in undergraduate and Masters level studies are sufficient for the work required. A PhD should remain optional.

\section{Rebuttal-Jeremy Booth}

It is notable that, while this debate is critically focused on the future, my opponent's case makes an argument focused on the past. It is true that TEAP registrars must keep an awareness of the past: indeed many radiotherapy departments still, today, do operate in the past. It is a past where physicists routinely stayed back late in the day after 'office hours' to undertake machine QA, where every patient plan required a chamber measurement, where physical wedges and blocks were commonplace, and where the traditional linear accelerator installation and commissioning took up to 3 months. It is a past absolutely founded in the tenants of quality management; of optimizing process controls and minimizing risk.

There is still a risk however, a potential failure mode that affects our very profession of physical scientists and engineers in medicine: pertaining to how we evolve to meet the challenge of automation and artificial intelligence that is pushing the field forward so quickly and undeniably. Do we become "end users" of fully locked down systems, or do we add value by channeling our expertise in highquality research that drives the high-quality systems we want to see?

TEAP, our vocational education program, is currently under review and must align with these current/future trends. Within a few years, future registrars will only know linear accelerators where daily QA is completed with a phantom and the EPID; or treatment planning systems where networked software performs secondary dose \& MU calculations on the fly, reducing PSQA measurements to nearzero. Even traditional linear accelerator commissioning is evolving. For example with the new Ethos (Varian Medical Systems, Palo Alto), installation takes just 1 week. Commissioning is mostly a spot check of the manufacturer provided beam model, adding just one more week. There is very little physical measurement required - or even possible—since the TPS essentially comes pre-commissioned, and the planning workflows are heavily automated. There are few problems here that an end user can solve.

My opponent talks about inconsistent hours in between routine work leading to poor research. On the one hand, this is really just a matter of time management. On the other: well, routine work is reducing, and perhaps "research" needs to become our new routine.

The accolades of the accredited physicist put by my opponent are not mutually exclusive to those with a $\mathrm{PhD}$. The PhD Physicist will be just as practical and hardworking. The PhD Physicist has that base of solid research experience, they innovate, they communicate and they synthesize complex multi-disciplinary ideas into safe and effective treatment. 
The scientific paradigm absolutely recognizes clinical excellence. Medical physics PhD students are embedded in the clinic solving real world problems. Let's reflect on Prof Jeraj, that eccentric after midnight dancing smiling guy, that my opponent references. I was on that early careers panel in 2016 and my opponent reports the findings correctly. But it was 2016! My fridge couldn't even tell itself to buy more milk back then. Prof Jeraj has been a lead component of the AAPM Medphys3.0 [13] movement working towards grand challenges-major research questions for medical physics, for $\mathrm{PhD}$ Physicists. Grand challenges stem from real world clinical problems, elevate them through innovation, collaboration and critical thinking to make significant improvements to human health.

The future of clinical medical physics will continue to innovate, automation will lead to reduction of clinical staff as it has in other professions, and our TEAP must adapt and possibly contract—by relying on incoming students being better prepared with postgraduate education. Radiotherapy physicists in the present will benefit from a $\mathrm{PhD}$, and those in the future will have one.

\section{Rebuttal-May Whitaker}

The skills and experience required of an accredited ROMP are not gained from a $\mathrm{PhD}$ alone. As the health sector becomes increasingly privatised, consideration of the bottom line will demand highly productive, multi-talented staff with skills beyond the cognitive. High potential employees will be strategic planners with strong business, financial and leadership skills, together with a high degree of emotional intelligence [14]. These are not normally developed during the course of a physics $\mathrm{PhD}$. Many universities offer additional courses to the $\mathrm{PhD}$ candidate in topics such as communication, networking and professional development, in a bid to render them more marketable to a future employer [15].

Dr Booth argues that the trend to employ fewer ROMPs is driven by the increasingly black box future of technology and the evolving role of the ROMP, moving away from process driven activities such as quality assurance checks and into more strategic and innovative pursuits. This, however, raises questions about the relevance of TEAP accreditation and affects both new and existing staff alike.

Retraining, retirement and redundancies will not necessarily drive an increase in $\mathrm{PhD}$ uptake. It may, in fact, result in an increase in computing, coding, analytics, business and management studies; skills which Dr Booth agrees are paramount in this black box future.

And whilst credential creep is a known phenomenon, it is the responsibility of individual managers to make the best hiring decision for their team and workplace. Cultural fit is often more important than number of degrees
[16]. No matter the credentials, if a new employee cannot fit into the team and workplace culture, years of training will not remedy the ensuing fracture of the team esprit de corps. Evidence based hiring practises should be used rather than overly focussing on the letters before or after one's name [17].

\section{References}

1. Moore K (2019) Automated treatment planning. Semin Radiat Oncol 29(3):209-218

2. Delaney G, Jacob S, Featherstone C, Barton M (2005) The role of radiotherapy in cancer treatment: estimating optimal utilization from a review of evidence-based clinical guidelines. Cancer 104(15):1129-1137

3. Glass P, Tracey E, Smetanin P, Kobak P, Pavlichev A, Bishop J (2008) Lives at Risk from Cancer in New South Wales 20072036. A health economics study of cancer in New South Wales. Cancer Institute NSW Monograph. https://www.cancer.nsw.gov. au. Accessed 20 May 2020

4. Herman MG, Harms TA et al (2008) Alternative clinical medical physics training pathways for medical physicists: Report of AAPM Task Group 133. https://www.aapm.org/pubs/reports/ RPT_133.pdf. Accessed 20 May 2020

5. Hamer B, Guilfoyle C (2019) Balancing technology, culture and supply. PwC Health Matters. PWC Australia. https://www. pwc.com.au/health/health-matters/workforce-healthcare.html. Accessed 20 May 2020

6. ACPSEM Position Statement. (2018) The role of physicists, Scientists and Engineers in medicine in Australasia. https://www. acpsem.org.au/as_agentcs.p?cmd=Document_AnywhereDo c(C0000020,00000055,6663bb366bdd4d1ecb16c660dbc294 227aba3bb5)\&TenID=ACPSEM. Accessed 20 May 2020

7. Pappano L (2011). The Master's as the New Bachelor's. The New York Times.pp 16-17. Accessed 20 May 2020

8. Why doing a PhD is often a waste of time. (2020) The Economist. https://medium.economist.com/why-doing-a-phd-is-often -a-waste-of-time-349206f9addb. Accessed 24 May 2020

9. Bourlan JD, Marsden DS, Hendee WR (2000) The Ph.D. degree is a handicap in the job market for clinical medical physicists. Med Phys 27(12):2641-2643

10. Doctor of Philosophy (Science) (2020) entry. University of Sydney. https://www.sydney.edu.au/courses/courses/pr/docto r-of-philosophy-science.html. Accessed 24 May 2020

11. Radiation Oncology Medical Physicists. (2020) ACPSEM. https ://www.acpsem.org.au/Meet-Our-Members/Radiation-Oncol ogy-Medical-Physicists. Accessed 24 May 2020

12. Jeraj R (2016) Early Careers Session. EPSM Sydney, 7-10 November

13. https://www.aapm.org/MedPhys30/articles/4304MedPhys30Re port.pdf Accessed 24 June 2020

14. Chamorro-Premuzic T, Adler S, Kaiser RB (2017). Harvard business review. what science says about identifying highpotential employees. https://hbr.org/2017/10/what-science-saysabout-identifying-high-potential-employees. Accessed 25 June 2020

15. Skills development. (2020) The University of Melbourne. https ://gradresearch.unimelb.edu.au/\#skills-development. Accessed 25 June 2020 
16. Bouton K. (2020). Harvard Business Review. Recruiting for cultural fit. https://hbr.org/2015/07/recruiting-for-cultural-fit. Accessed 25 June 2020

17. Is credential creep strangling your hiring. (2020) Talent Management \& HR. https://www.tlnt.com/is-credential-creep-strangling -your-hiring/. Accessed 25 June 2020
Publisher's Note Springer Nature remains neutral with regard to jurisdictional claims in published maps and institutional affiliations. 